\title{
Morphology of SNRs and their halos
}

\author{
R. Brose, ${ }^{a, b, *}$ M. Pohl ${ }^{b, c}$ and I. Sushch ${ }^{d, e}$ \\ ${ }^{a}$ Dublin Institute for Advanced Studies \\ 31 Fitzwilliam Place, Dublin 2, Ireland \\ ${ }^{b}$ Institute of Physics and Astronomy, University of Potsdam \\ 14476 Potsdam, Germany \\ ${ }^{c}$ DESY, 15738 Zeuthen, Germany \\ ${ }^{d}$ Centre for Space Research, North-West University, 2520 Potchefstroom, South Africa \\ ${ }^{e}$ Astronomical Observatory of Ivan Franko National University of Lviv, Kyryla i Methodia 8 , \\ 79005 Lviv, Ukraine \\ E-mail: robert.brose@desy.de
}

We use our time-dependent acceleration code RATPaC to study the formation of extended gammaray halos around supernova remnants and the morphological implications that arise when the high-energetic particles start to escape from the remnant.

We performed spherically symmetric 1-D simulations in which we simultaneously solve the transport equations for cosmic rays, magnetic turbulence, and the hydrodynamical flow of the thermal plasma in a volume large enough to keep all cosmic rays in the simulation.

For older supernova remnants we find strong morphological differences between the hadronic and the leptonic gamma-ray intensity. At early times both - the inverse-Compton and the Piondecay morphology - are shell-like. However, as soon as the maximum-energy of the freshly accelerated particles starts to fall, the inverse-Compton morphology starts to become centerfilled whereas the Pion-decay morphology retains its shell-like structure. Escaping high-energy electrons start to produce an emission halo around the remnant at this time. There are good prospects for detecting the spectrally hard halo component with the future Cherenkov Telescope Array, likewise for detecting variations of the gamma-ray spectral index across the interior of the remnant, whereas current-generation gamma-ray observatories have insufficient sensitivity. Further, we find a constantly decreasing non-thermal X-ray flux that makes a detection of X-ray unlikely after the first few thousand years of the remnants evolution. The radio flux is increasing throughout the SNR's lifetime and changes from a shell-like to a more center-filled morphology later on.

$37^{\text {th }}$ International Cosmic Ray Conference (ICRC 2021)

July 12 th - 23rd, 2021

Online - Berlin, Germany

\footnotetext{
*Presenter
} 


\section{Introduction}

A self-regulation of the diffusion coefficient by escaping particles is known to exist around supernova remnants (SNRs). There, the scattering turbulence is created mainly by escaping hadrons, and the electrons accelerated at the SNR blast-wave are trapped as a side effect [19,20].

So far, there has not been a direct measurement of CRs escaping from a SNR. There is evidence of larger extension of the gamma-ray emission around RX J1713 compared to the X-ray emission that could indicate CR escape [11]. However, there is indirect evidence that CR escape has to happen around SNRs. Recent studies of particle acceleration in supernova remnants showed that the typical soft, broken power-law spectra of aged SNRs [29] result from the escape of high-energy particles from the interior of the SNR. Here, the most energetic particles escape once the SNR is no longer capable of accelerating them any further, creating soft spectra inside the SNR where most of the emission is produced [5, 7].

\section{Basic equations and assumptions}

The methodology is similar to that of [5], and here we shall only give a short summary. We combine a kinetic treatment of the CRs with a thermal leakage injection model, a fully time-dependent treatment of the magnetic turbulence, and a PLUTO-based simulation of the hydrodynamical flow profiles.

\subsection{Cosmic rays}

We solve the kinetic equation for the differential number density of cosmic rays, $N$,

$$
\frac{\partial N}{\partial t}=\nabla\left(D_{r} \nabla N-\mathbf{u} N\right)-\frac{\partial}{\partial p}\left((N \dot{p})-\frac{\nabla \cdot \mathbf{u}}{3} N p\right)+Q
$$

in the test-particle limit, where $D_{r}$ denotes the spatial diffusion coefficient, $\mathbf{u}$ the advective velocity, $\dot{p}$ energy losses, and $Q$ the source of thermal particles [24]. We use a thermal leakage model $[3,16]$ for the injection of particles. This injection scenario is a simplification, in particular for electrons, for which pre-acceleration to a few tens of $\mathrm{MeV}$ is required and established at the shock $[4,14,17]$. We are interested in particles at energies well above $100 \mathrm{MeV}$, and so the particulars of that pre-acceleration can be ignored. For this work we choose injection parameters that guarantee a $\mathrm{CR}$ pressure of less than $2.5 \%$ of the shock ram pressure during the entire simulation. The number of injected particles is close to that seen in SN 1006.

In addition to the synchrotron losses, electrons will also suffer losses by collisions with photons from background photon-fields. Usually these inverse-Compton (IC) losses can be neglected as synchrotron losses in amplified magnetic field will dominate. However, high-energy electrons spend a large fraction of time upstream of the shock, where IC and synchrotron losses are of similar strength. We used the approximations derived by [22] to account for IC losses by collisions with the cosmic microwave background.

\subsection{Magnetic turbulence}

In parallel to the transport equation for CRs, we solve a transport equation for the magneticturbulence spectrum, assuming Alfvén waves only. The temporal and spatial evolution of the 
spectral energy-density per unit logarithmic bandwidth, $E_{w}$, is described by

$$
\frac{\partial E_{w}}{\partial t}+\cdot \nabla\left(\mathbf{u} E_{w}\right)+k \frac{\partial}{\partial k}\left(k^{2} D_{k} \frac{\partial}{\partial k} \frac{E_{w}}{k^{3}}\right)=2\left(\Gamma_{g}-\Gamma_{d}\right) E_{w} .
$$

Here, $\mathbf{u}$ denotes the advection velocity, $k$ the wavenumber, $D_{k}$ the diffusion coefficient in wavenumber space, and $\Gamma_{g}$ and $\Gamma_{d}$ the growth and damping terms, respectively [6].

We use a growth-rate based on the resonant streaming instability [1, 24],

$$
\Gamma_{g}=A \cdot \frac{v_{\mathrm{A}} p^{2} v}{3 E_{\mathrm{w}}}\left|\frac{\partial N}{\partial r}\right|
$$

where $v_{\mathrm{A}}$ is the Alfven-velocity. We introduced a linear scaling factor, $A$, to artificially enhance the amplification. We used $A=10$ throughout this paper to mimic the more efficient amplification due to the non-resonant streaming instability $[2,15]$. The value we choose for $A$ guarantees a cut-off energy in the gamma-ray spectrum between $1-10 \mathrm{TeV}$ as observed in young SNRs.

We calculate the total magnetic-field strength as $B_{\text {tot }}=\sqrt{B_{0}^{2}+B_{\text {Turb }}^{2}}$, where $B_{0}$ is the large-scale magnetic field. We solve the induction equation to model the transport of the frozen-in large-scale magnetic field [26]. The far-upstream field is assumed to be uniform with strength $5 \mu \mathrm{G}$.

Since we exceed the growth rate of the resonant streaming instability [1] by a factor of ten, the turbulent field is amplified to $\delta B \gg B_{0}$ during the initial phases of SNR evolution.

The growth of the magnetic turbulence and hence the magnetic field is balanced by cascading. This process is described as a diffusion process in wavenumber space, and the diffusion-coefficient is given by $[23,30]$

$$
D_{\mathrm{k}}=k^{3} v_{\mathrm{A}} \sqrt{\frac{E_{\mathrm{w}}}{2 B_{0}^{2}}} .
$$

This phenomenological treatment will result in a Kolmogorov-like spectrum, if cascading is dominant.

\subsection{Thermal plasma}

In the test-particle limit, the evolution of an SNR can be described with the standard gasdynamical equations. We solve the hydro-equations under the assumption of spherical symmetry in 1-D using the PLUTO code [18]. The non-equilibrium cooling function, $L$, is taken from [25].

In this work, we display results for type-Ia supernova explosions. We initiate the simulations with exponential-ejecta profiles:

$$
\begin{aligned}
& \rho_{\mathrm{SN}}=A \exp \left(-v / v_{e}\right) t_{i}^{-3,} \text { and } v=r / t_{i}, \\
& \text { with } v_{e}=\left(\frac{E_{\mathrm{ex}}}{6 M_{\mathrm{ej}}}\right)^{1 / 2} \text { and } A=\frac{6^{3 / 2}}{8 \pi} \frac{M_{\mathrm{ej}}^{5 / 2}}{E_{\mathrm{ex}}^{3 / 2}}
\end{aligned}
$$

as initial conditions [8]. Here, $t_{i}=2.5 \mathrm{yrs}$ is the start time of our simulation, $M_{\mathrm{ej}}=1.4 M_{\mathrm{sol}}$ the ejecta mass, $E_{\mathrm{ex}}=10^{51} \mathrm{erg}$ the explosion energy, and $r$ the spatial coordinate. The density of the ambient medium was chosen to be $0.4 \mathrm{~cm}^{-3}$. 

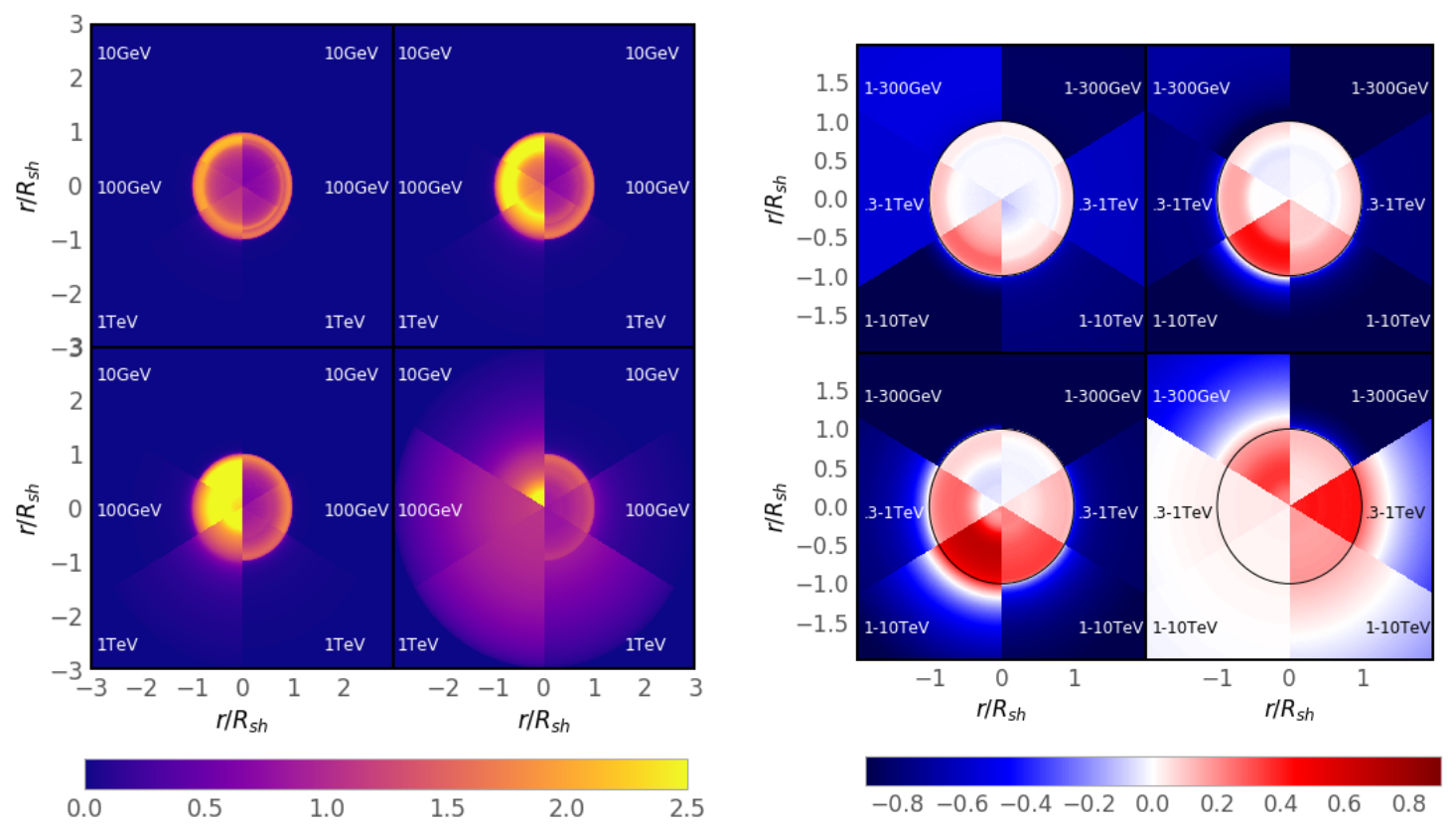

Figure 1: Left panel: Maps of normalized surface brightness of a Type-Ia SNR after 300, 1000, 2,000 and $10,000 \mathrm{yrs}$ (from top left to bottom right). Left hemispheres are for IC emission and right hemispheres for PD emission. Right panel: Maps of spectral-index variation in three energy bands at 300, 1000, 2,000 and $10,000 \mathrm{yrs}$ (from top left to bottom right), relative to the average spectral index of the remnant (including the halo). Left hemispheres are inverse-Compton emission and right hemispheres Pion-decay emission.

\section{Results}

We followed the evolution of the remnant for 25, 000 years. The total magnetic field strength reaches $90 \mu \mathrm{G}$ after $300 \mathrm{yrs}$ and drops to $40 \mu \mathrm{G}$ after $1000 \mathrm{yrs}$. After 10, $000 \mathrm{yrs}$, the turbulent field is weaker than the compressed large-scale field.

\subsection{Halo and spectral index evolution}

We calculated intensity maps for IC and PD emission at three energies over the lifetime of the SNR. The results are presented in Figure 1.

Initially, both radiation mechanisms produce a shell-like morphology in all energy bands. From roughly 1000 years on, the shell thickness of IC emission exceeds that of PD radiation, because the latter is boosted by the high gas density immediately downstream of the shock. Contrary, the IC emission reflects only the distribution of electrons, and already after 1000 years - the onset of the Sedov-stage - we notice IC emission outside the SNRs shell. The transition to the Sedov-phase usually also marks the time of the highest maximum energy of particles [21]. At later times, particles of the highest energy start to escape from the remnant [5].

Even after 10,000 years, the PD morphology remains shell-like whereas that of IC emission becomes center-filled. Even low-energy CRs propagated into the center of the remnant, and the projection enhances the brightness towards the center of the remnant in the IC channel. The low density of the thermal plasma in the center keeps suppressing PD emission from the central region. 
As more and more high-energy electrons escape with increasing age, an extensive halo of $>100$ $\mathrm{GeV}$ electrons is formed around the remnant. The halo in the PD channel remains faint even after 10,000 years on account of the low gas density upstream of the forward shock.

The angular resolution of gamma-ray observatories is key to a study of spatial variations of the gamma-ray spectra [11-13].

The spectrum of emission from outside the remnant is typically harder than that from the interior. Figure 1 illustrates spectral index variations across the remnant at different ages. For emission from the interior of the remnant, the deviations are moderate, $|\delta s| \lesssim 0.2$, in all three energy bands that we consider, but those of the halo emission can be larger.

The detection of hadronic halo emission is unlikely given the current observational sensitivities of $\Delta s \approx \pm 0.1-0.2$. Considering the limited spatial resolution and the poor statistics in some regions, a significant detection of spectral-index deviations is unlikely with current-generation IACTs. The higher sensitivity of CTA should reduce the uncertainty, $\Delta s$ and possibly allow the detection of spectral-index variations.

\subsection{Reduction of the diffusion coefficient}

The escape of particles from the SNR and consequently the amplification of turbulence change the diffusion coefficient in the vicinity of the SNR [9, 10]. Figure 2 shows that spatial variation relative to the assumed diffusion coefficient in the ISM, which is set to $10 \%$ of the conventional Galactic diffusion coefficient [27].

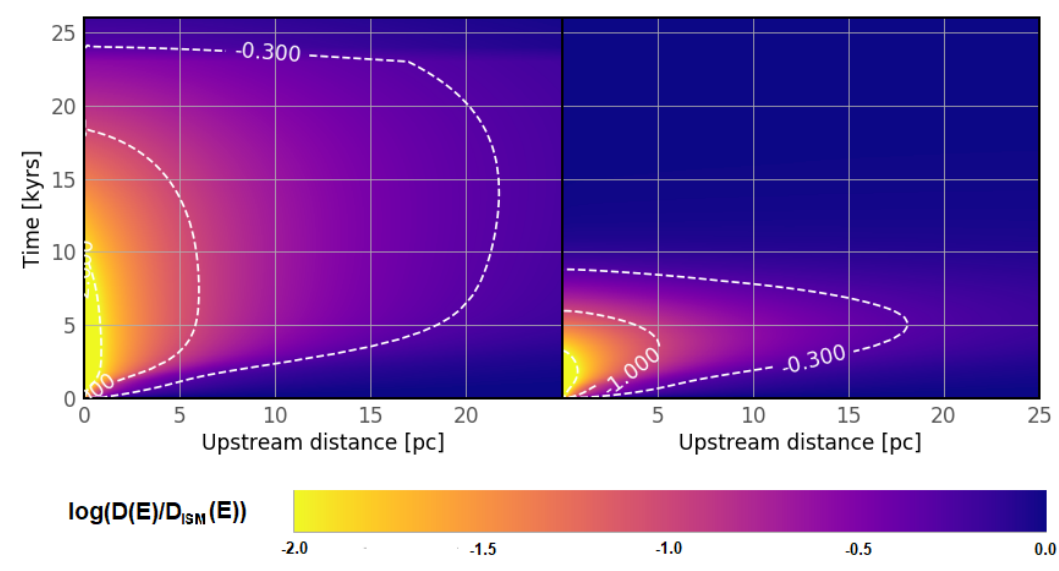

Figure 2: Normalized diffusion coefficient in the upstream region as function of distance to the shock and the SNR age. The left panel is for a particle energy of $100 \mathrm{GeV}$, the right panel for $3 \mathrm{TeV}$. The dashed lines correspond to $1 \%$, $10 \%$ and $50 \%$ of $D_{\text {ISM }}$.

For both electrons and ions the reduction of the diffusion coefficient is strongest after 5, 000 years (10\%-level) and 2,000 years (1\%-level), around the time of, or shortly after, the transition from free expansion to the Sedov-Taylor phase, when the maximum energy of the accelerated particles starts to decrease. Then there are too few freshly accelerated particles at high energy to sustain the level of turbulence, and consequently the diffusion coefficient starts to increase. The main damping mechanism of turbulence is cascading. Consequently, the distributions in Figure 2 show an energy-dependent time-evolution. The spatial extent is similar though, because the suppression of the diffusion coefficient for $100 \mathrm{GeV}$-particles is governed mainly by cascading from smaller $k$, i.e. by turbulence driven by particles at higher energy. Turbulence driving by the low-energy particles is only important very close to the shock. 


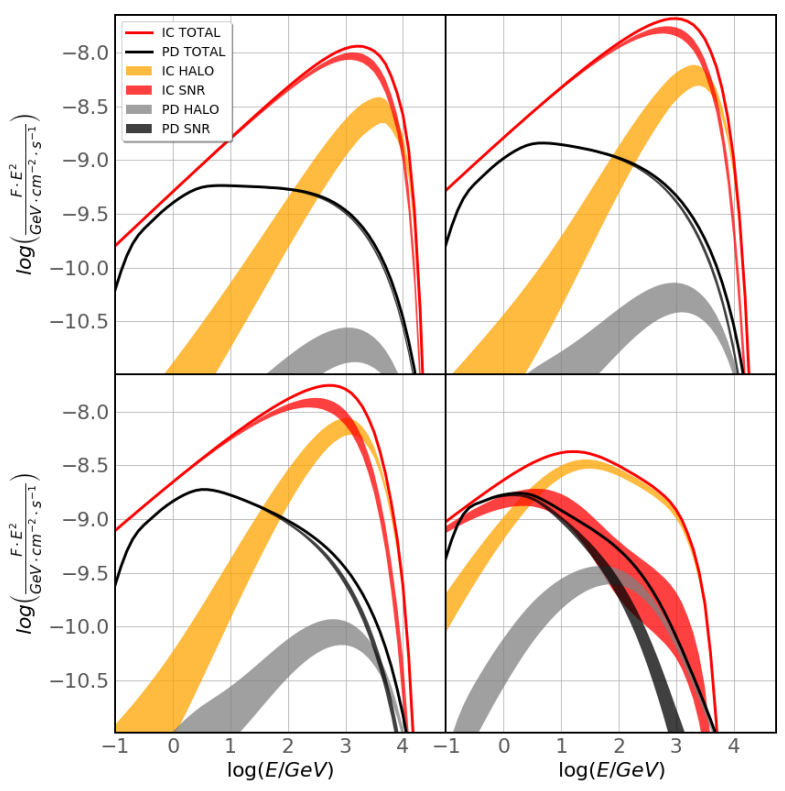

Figure 3: Comparison of emission spectra for IC (red) and PD (black) emission. Emission from the SNR is filledblack and filled-red for PD and IC-emission respectively. The halo-emission is filledorange and filled-gray for IC and PDemission respectively at $300,1000,2,000$ and 10,000yrs (from top left to bottom right). The spectra including (and excluding) the projection effect constitute the upper (lower) boundaries of the SNR emission and the lower (upper) limit of the area for emission from the halo.

During the initial growth of turbulence in the precursor, for the two particle energies shown in Figure 2 the diffusion coefficient shows the same trend, until the supply of freshly accelerated $3-\mathrm{TeV}$ particles is exhausted. However, the precursor scale of $100-\mathrm{GeV}$ particles is significantly smaller than for $3-\mathrm{TeV}$ particles, because the turbulence scattering the lower-energy particles results from cascading of modes resonant with the more energetic particles. Later, the escape of particles, that were trapped in the interior of the remnant, becomes the main driver of turbulence, and in fact the maximum extent of the turbulence precursor, and hence the reduced diffusion coefficient, is reached well after the beginning of the Sedov-phase.

\subsection{Detectability}

Figure 3 shows the emission spectra expected from the SNR itself and the halo at different times for a generic distance of $1 \mathrm{kpc}$ to the remnant. The contribution from the halo is shown twice, once as volume-integrated emission from $r>r_{s h}$, and once only the component that in projection appears to come from outside of the SNR.

The evolution of the spectral energy distributions (SEDs) for PD-emission clearly shows the mechanism for the spectral softening [5] for both emission channels. The lack of target material outside the SNR suppresses hadronic emission from the halo at all times. The halo emission from IC scattering is always much brighter than the PD component. Overall, the flux from the halo is $20 \%$ to $30 \%$ of that of the SNR itself, making a detection possible only for the brightest known Galactic SNRs. As low-energy particles reside closer to the shock, they contribute little to the projected halo emission whose spectrum is thus relatively hard.

Leptonic emission detected from an SNR by current-generation gamma-ray observatories probably includes emission from the halo. Most of it is produced close to the remnant and in projection is very difficult to observationally distinguish. What may have been seen already in RXJ1713.7-3946 is that the leptonic halo causes a larger extent of the very-high gamma-ray emission than that of the X-ray emission. 

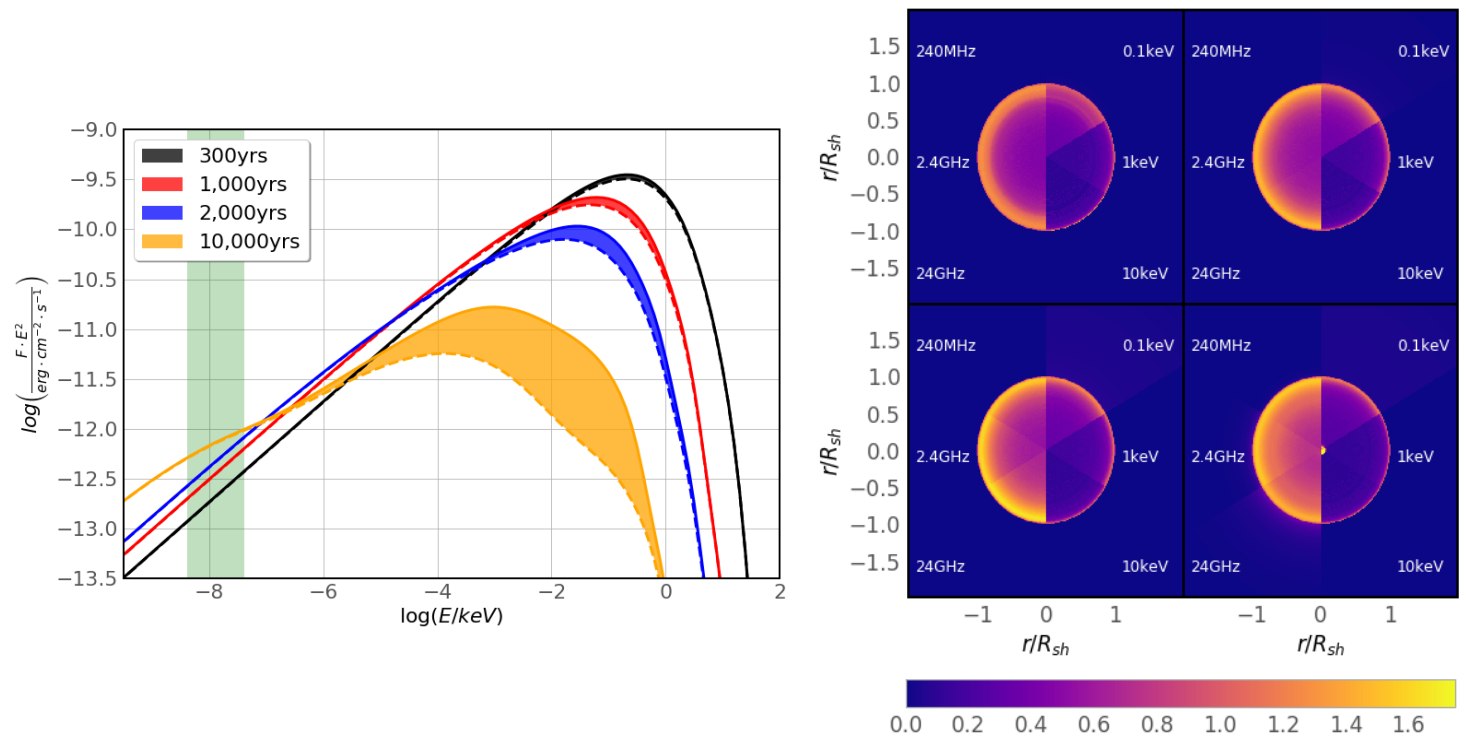

Figure 4: Left panel: Synchrotron flux from the remnant at various times. The upper boundaries of the filled areas mark the total emission from the remnant while the lower boundaries indicate emission from downstream only. The green band indicates the $1-10 \mathrm{GHz}$ range. Right panel: Normalized synchrotron intensity from a Type-Ia SNR after 300, 1000, 2,000 and 10,000 yrs (from top left to bottom right).

\subsection{Synchrotron emission}

We evaluated the non-thermal synchrotron emission from the SNR over its lifetime (Figure 4). Contrary to the IC-emission, there is little difference between the total and the downstream-only flux, except for very old remnants, because a strong magnetic field boosts the emission from the downstream region. At later times, most of the high-energy electrons were able to escape the remnant and the magnetic field is not amplified any more, the halo emission starts to dominate.

The flux in the X-ray band decreases with time, because the magnetic-field strength decreases and cooling takes its toll. Consequently, the remnant is brightest and best detectable during the first 2, 000 yrs after the supernova - matching the census of Galactic SNRs [28].

In contrast, the radio flux increases throughout the entire lifetime of the SNR. The steady accumulation of $\mathrm{GeV}$-scale particles compensates for the weakening magnetic field. Interestingly, the escape of electrons affects the spectral index in the radio band. Between $1 \mathrm{GHz}$ and $30 \mathrm{GHz}$ (Roughly $10^{-8} \mathrm{keV}$ to $10^{-7} \mathrm{keV}$ ) the radio spectra are fairly soft after $10,000 \mathrm{yrs}$, with a spectral index $\alpha \approx 0.75\left(S_{v} \propto v^{-\alpha}\right)$, close to that observed for very old SNRs.

Figure 4 shows the emission morphology at radio and X-ray energies for four stages of SNR evolution. Throughout the SNR's lifetime the magnetic-field strength peaks right downstream of the forward shock. Consequently, the synchrotron emissivity peaks immediately downstream to the shock-location. The radio emission shows the same shell-like structure. However, the spatial variation of the magnetic field has a moderate impact on the synchrotron emissivity, typically $\propto B^{1.5}$. The radio shells appear thicker as a consequence. Moreover, diffusion of electron towards the center of the remnant partially compensates the weak magnetic field in the interior. Thus, the remnant appears somewhat center-filled in the radio band at later stages. 


\section{Conclusions}

We performed numerical simulations of particle acceleration in SNRs, solving time-dependent transport equations of CRs and magnetic turbulence in the test-particle limit alongside the standard gas-dynamical equations for Type-Ia SNRs. We derived the CR diffusion coefficient from the spectrum of magnetic turbulence, that evolves through driving by the resonant streaming instability as well as cascading and wave damping.

- Leptonic gamma-ray emission tends to produce a center-filled appearance with increasing remnant age

- Hadronic gamma-ray emitters retain a shell-like morphology

- The detection of a leptonic gamma-ray halo around SNRs is in sight with CTA

- There is strong spatial and temporal evolution of the diffusion coefficient in the upstream of the shock. Diffusion of low-energetic particles is gonverend by down-cascading of turbulence from larger scales

- The deviation of the spectral index in the gamma-ray domain by $\Delta s \approx 0.2$ is likely not detectable with current generation IACTs and might be marginally detected by CTA

- The non-thermal X-ray emission cedes after a few thousand years and the radio-morphology of the remnant becomes more center-filled. A softening in the radio-band is observed at the latest stages.

\section{References}

[1] Bell, A. R. 1978, MNRAS, 182, 147

[2] Bell, A. R. 2004, MNRAS, 353, 550

[3] Blasi, P., Gabici, S., \& Vannoni, G. 2005, MNRAS, 361, 907

[4] Bohdan, A., Niemiec, J., Pohl, M., et al. 2019, ApJ, 878, 5

[5] Brose, R., Pohl, M., Sushch, I. Petruk, O., \& Kuzyo, T. 2020, A\&A, 634, A59

[6] Brose, R., Telezhinsky, I., \& Pohl, M. 2016, A\&A, 593, A20

[7] Celli, S., Morlino, G., Gabici, S., \& Aharonian, F. A. 2019, MNRAS 490,4317

[8] Dwarkadas, V. V. \& Chevalier, R. A. 1998, ApJ, 497, 807
[9] Fujita, Y, Ohira, Y \& Takahara, F. 2010, ApJL, 712, L153

[10] Fujita, Y., Takahara, F., Ohira, Y., \& Iwasaki, K. 2011, MNRAS, 415, 3434

[11] H. E. S. S. Collaboration, Abdalla, H., Abramowski, A., et al. 2018, A\&A, 612, A6

[12] H. E. S. S. Collaboration, Abdalla, H., Abramowski, A., et al. 2018, A\&A, 612, A7

[13] Humensky, B. \& VERITAS Collaboration 2015, in International Cosmic Ray Conference, Vol. 34, 34th International Cosmic Ray Conference (ICRC2015), 875

[14] Li, R., Zhou, C. T., Huang, T. W., et al. 2018, Physics of Plasmas, 25, 082103 MNRAS, 314,65

[16] Malkov, M. A. 1998, Physical Review E, 58, 4911

[17] Matsumoto, Y., Amano, T., Kato, T. N., \& Hoshino, M. 2017, Phys Rev. Lett.

[18] Mignone, A., Bodo, G., Massaglia, S., et al. 2007, ApJS, 170, 228

[19] Nava, L., Gabici, S., Marcowith, A Morlino, G., \& Ptuskin, V. S. 2016, MNRAS, 461,3552

[20] Ohira, Y., Murase, K., \& Yamazaki, R. 2010, A\&A, 513, A17

[21] Ptuskin, V. S. \& Zirakashvili, V. N. 2003, A\&A, 403,

[22] Reimer, A., Pohl, M., \& Reimer, O. 2006, ApJ, 644, 1118
[23] Schlickeiser, R. 2002, Cosmic Ray Astrophysics

[24] Skilling, J. 1975, MNRAS, 172, 557

[25] Sutherland, R. S. \& Dopita, M. A 1993, ApJS, 88, 253

[26] Telezhinsky, I., Dwarkadas, V. V. \& Pohl, M. 2013, A\&A, 552, A102

[27] Trotta, R., Jóhannesson, G., Moskalenko, I. V., et al. 2011, ApJ, 729, 106

[28] Vink, J. 2012, A\&A Rev., 20, 49

[29] Zeng, H., Xin, Y., \& Liu, S. 2019 , ApJ, 874, 50

[30] Zhou, Y. \& Matthaeus, W. H. 1990 Journal of Geophysics Research, 95,14881 